\title{
Hybrid intelligence in business networks
}

\author{
Philipp Ebel $^{1} \cdot$ Matthias Söllner $^{2} \cdot$ Jan Marco Leimeister ${ }^{1,3} \cdot$ Kevin Crowston $^{4} \cdot$ Gert-Jan de Vreede $^{5}$
}

Published online: 11 June 2021

(C) Institute of Applied Informatics at University of Leipzig 2021

\section{Introduction}

In the field of computer science, as well as in the information systems domain, artificial intelligence constitutes one of the most rapidly growing streams of research (Jordan \& Mitchell, 2015). Interest is rising because new technological innovations enable the development of productive artificial intelligence solutions that provide compelling benefits in various fields of application. Artificial intelligence systems have reached a level of productivity where they do have the potential to reduce business costs, enhance business analytics, and ultimately improve the quality and effectiveness of managerial decisions. As a consequence, artificial intelligence has evolved from a field of research to a viable technology that is used in a wide array of application fields (Jordan \& Mitchell, 2015). In fact, artificial intelligence

Philipp Ebel

philipp.ebel@unisg.ch

Matthias Söllner

soellner@uni-kassel.de

Jan Marco Leimeister

janmarco.leimeister@unisg.ch

Kevin Crowston

crowston@syr.edu

Gert-Jan de Vreede

gdevreede@usf.edu

1 Institute of Information Management, University of St. Gallen, Müller-Friedberg-Strasse 8, 9000 St.Gallen, Switzerland

2 Research Center for IS Design (ITeG), Information Systems and Systems Engineering, University of Kassel, Henschelstr. 4, 34127 Kassel, Germany

3 Resarch Center for IS Design (ITeG), Chair for Information Systems, University of Kassel, Pfannkuchstrasse 1, 34121 Kassel, Germany

4 School of Information Studies, Syracuse University, Hinds Hall, Syracuse, NY 13244-4100, USA

5 Muma College of Business, University of South Florida, 4204 E. Fowler Av., Tampa, FL 33629, USA developments are setting the stage for a paradigm shift in the workplace: According to PwC, $72 \%$ of business executives say that artificial intelligence will give them a competitive advantage in the future (Bothun et al., 2017). Many repetitive and simple tasks that were typically performed by humans are now being automated by artificial intelligence bundled with other technologies, such as cloud systems, augmented reality, (social) robots, IoTs, and wearables. As a result, the ratio of tasks executed by machines to humans is about to shift dramatically (WEF, 2020). As a consequence, there is a broad consensus that artificial intelligence has the potential to deliver huge economic benefits (Jordan \& Mitchell, 2015).

Despite these recent advances, artificial intelligence systems still exhibit major deficits (Attenberg et al., 2015). Without the ongoing supervision of humans, systems can make mistakes or fail completely. For example, chatbots, a form of conversational agents - a subset of artificial intelligence-if poorly designed, can be as biased as humans. Recently, a Microsoft chatbot called "Tay" was made into a racist, sexist and antisemitic chatbot in less than $24 \mathrm{~h}$ after being available for interactions with real users online. This highlighted chatbots' vulnerability to manipulation. In addition to that, when learned models are incomplete, there is a mismatch between training and test distributions, or rare cases such as outliers might be both highly informative and relevant (Lakkaraju et al., 2017). Especially when it comes to critical tasks such as driver assistance or medical diagnosing, failures of these systems will have serious implications on the well-being of humans, thus resulting in a negative impact of the users' trust towards the system.

To address these challenges, recent research in the field focuses on the design of so-called Hybrid Intelligence Systems (HIS), characterized by a close partnership between humans and machines. In this partnership, different facets, such as task specification, creation of incentives, task allocation, quality assessment, task aggregation, and compensation mechanisms have to be considered. In that, HIS constitute a special form of digital platforms, in which socio-technical interactions are coordinated virtually (Kenney \& Zysman, 2016). Articles have concentrated on the technological 
issues that are related to the development of HIS and highlight the novel character of this concept (Cheng \& Bernstein, 2015; Kamar, 2016a, b). Additional insights into the relationships between design decisions, actors' behavior, and business outcomes therefore constitute a promising avenue for further research that deserves to be addressed by researchers and practitioners. The time is now to call for theoretical and empirical underpinnings on how hybrid intelligence can be utilized. As Electronic Markets contributed significantly to developing our understanding on how other kinds of artificial intelligence systems generate value within business networks (Dellermann et al., 2019b; Kühl et al., 2020; Neuhofer et al., 2020), we are glad that, in this tradition, we can bundle papers on a relevant topic: Hybrid Intelligence in Business Networks.

\section{Positioning hybrid intelligence in IS literature}

Instead of focusing on the development of a general artificial intelligence, recent research in the field focuses on the design of HIS (Dellermann et al., 2019a). A hybrid system allows the integration of human input into an artificial intelligence system throughout the system's life cycle in order to develop, complement, and evaluate the system's capabilities. By combining the complementary strengths of human intelligence and artificial intelligence both will behave more intelligent than each of the two could be in separation (Kamar, 2016a, b). In this regard, the human input can help an artificial intelligence system to avoid mistakes and the human feedback can be used to realize optimized learning cycles as the machine is able to continuously learn from its human partner. In that, the issue is not which is 'better', the issue is how you can create synergy between human intelligence and artificial intelligence so that they outperform the separate entities together as a team/symbiotic entity. Today, most artificial intelligence systems benefit from human input only during the development or training cycles. In these phases, the input of software developers and experts is used in order to train the system. However, once deployed within the learning cycle, human input that the system can use in order to improve its decisions is often minimal. But without ongoing human input, the performance of the system will not be able to overcome biases and limitations that have been implemented in the course of the system development (Engel et al., 2021 a, b) and indeed, performance may decrease continuously due to the changing parameters of the system's tasks.

Human input can be used to help a system avoid mistakes and to realize optimized learning cycles as the machine is able to continuously learn from its human partner. In that, the issue is not which kind of intelligence is 'better': the issue is how to create synergy between human intelligence and artificial intelligence so that they outperform the separate entities together as a team/symbiotic entity. Against this backdrop, hybrid intelligence aims at the combination of complementary heterogeneous intelligences (i.e. human and artificial agents) into a socio-technological ensemble that is able to overcome the current limitations of (artificial) intelligence (Engel et al., 2021a, b). This approach is neither focusing on human intelligence in the loop of artificial intelligence nor automating simple tasks through machine learning. Rather, the emphasis lies on solving complex problems using the deliberate allocation of tasks among different heterogeneous algorithmic and human agents. Both the human and the artificial agents of such systems can then co-evolve by learning and achieve a superior outcome on the system level.

\section{Research streams in hybrid intelligence literature}

As of now, research in the field of hybrid intelligence can be clustered along three different streams.

\section{Development of hybrid intelligence systems}

When it comes to designing HIS, research in the field examined how the state of a system as well as its input requirements can be presented in a way that human helpers can understand and process them accordingly. In HIS, this problem corresponds to the challenge of task design to elicit high quality work from the human helpers. For example, Wang et al. (2012) explored different task designs to collect semantic input so that the results can be used in order to train the system for language understanding. In another study, Mitchell et al. (2014) investigated how human helpers can participate in the language generation process of a spoken dialog system. A second stream of research, examines how the noise of the input of human helpers affects interactions between humans and machines. Ipeirotis (2010) developed algorithmic models that are able to learn about the expertise and knowledge of human workers. These models are able to correct individual mistakes as long as the individual noise of the workers is independent. However, when a specific bias can be found in a whole population of workers, these systems may fail when trying to achieve a certain task (Kamar et al., 2015). As a consequence, existing research has identified the need for models that can successfully learn about the task-dependent biases and correct them. Third, research has examined the fluidity of human capabilities. While computational input that is used in artificial intelligence systems can only be used for a predefined training scenario, human input can be extended to a wide range of 
tasks. Consequently, studies have examined whether human helpers can acquire new capabilities that are used within different training tasks. Doroudi et al. (2016) performed a series of controlled studies to measure the effectiveness of different training strategies for improving the performance of human helpers in different settings. These training strategies included expert examples, which require additional work from a domain expert, and peer validation, which asks workers to validate the work of their peers as a form of training.

\section{Design of hybrid intelligence systems}

The second research stream covers the design of the collaboration process between humans and machines. In the context of collaborative processes, machine-learning-based systems could not only actively participate in solving given problems, but also act as facilitators within the collaboration process (Seeber et al., 2020). For example, Smart Personal Assistants (SPAs) are expected to increase the productivity of workers, by adapting to their tasks and their individual working routines (Knote et al., 2019, 2021). Within private environments, SPAs are designed to increase the user's wellbeing and comfort. As a consequence, SPAs are becoming increasingly popular in both domains. However, due to the high amount of contextualization that is needed to provide a flawless user experience, creation of a consistent collaboration process between human and system is challenging for designers (Maedche et al., 2019). As a result of these simultaneous design efforts, conventional approaches towards interaction design often fall short, as design iterations based on prototyping and user testing are difficult to realize (Lee et al., 2017). As a result, previous research has provided different design approaches for the development and contextualization of comprehensive SPAs for a variety of hybrid scenarios (Feine et al., 2019; Følstad \& Brandtzæg, 2017; Wambsganss et al., 2020; Winkler et al., 2020a, b).

In addition, research in this stream also investigates how new forms of human-computer interactions with conversational interfaces using natural language, either written or spoken, could evolve (Poser \& Bittner, 2020). Nowadays, conversational interfaces such as Apple's Siri, Microsoft's Cortana, Amazon's Alexa, or Google's Assistant are ubiquitous, and they are used in several areas, such as customer service, health care or education (Janssen et al., 2020). For example, research by Winkler et al. (2020b), Wambsganss et al. (2021) and (Winkler et al. 2021) has shown that the use of SPAs in educational settings has a positive impact on different learning outcomes, such as problem solving or argumentation skills. Further, research in this stream also analyzes how HIS can be employed to foster innovation in various value creation processes (Elshan \& Ebel, 2020; Elshan et al., 2021; Engel \& Ebel, 2019).

\section{Management of hybrid intelligence systems}

When analyzing research and practice in the field of HIS, it becomes evident that there are frequent examples in which systems do not achieve the desired results. Reasons for these failures can be found in all elements of a HIS. Due to a lack of expertise, the human partners may not be able to provide the desired input or deliberately deliver wrong results due to insufficient motivation. On the artificial intelligence side, there are examples in which the system is unable to evaluate the delivered input correctly and fails to integrate it into its own decision-making processes. Finally, human partners are not provided with a suitable platform that enables them to perform a task quickly and easily, or the workflows on the platform are failing to create incentive structures that motivate and engage human helpers without unintentionally affecting their performance.

Current research has addressed these issues by examining how the noise of the input of human helpers is affecting interactions between humans and machines (Ipeirotis, 2010) or whether human assistants can acquire new skills by participating in certain training tasks (Doroudi et al., 2016), as mentioned above. Also, there are studies that investigate how different incentive structures are influencing the trade-offs in quality and effort in HIS (Mao et al., 2013). Moreover, research has examined technological features for allocating resources when assigning workers for certain tasks (Kamar et al., 2012).

Finally, the transfer of certain degrees of cognition from humans to machines and the increasing rise of tasks that are amenable to cognitive automation (Engel et al., 2021a, b) will also have a huge impact on the future of work (Card \& Nelson, 2019). This circumstance will require organizations to adapt structures and organizational practices (Engel et al., $2021 \mathrm{a}, \mathrm{b})$ and align the new technology with a comprehensive strategy regarding the future of work (Zarkadakis et al., 2016). Here, hybrid intelligence will become increasingly important, as there will be an increasing number of tasks and processes that are performed neither purely by humans nor purely by cognitive machines, and where symbiotic entities - the combination of human and machine agents-achieve higher performance together than when they act separately (Dellermann et al., 2019a; Engel et al., 2021a, b).

\section{Papers in this special issue}

Although a considerable amount of exploration has been conducted regarding the collaboration between human intelligence and artificial intelligence, the breadth and scope for dialogue and experimentation needs to be broadened. This special section provides a place for such dialogue and support of a diverse community interested in taking the 
challenge further. The three papers that have been selected follow the outlined research streams and provide an excellent starting point for further research on hybrid intelligence.

The first paper, "Human-Machine Collaboration in Online Customer Service - a Long-Term Feedback-Based Approach" by Roland Graef, Mathias Klier, Kilian Kluge and Jan Felix Zolitschka (2021), deals with the rising expectations of customers, who expect to be able to contact a company via e-mail, chat, and social media platforms, while demanding ever shorter response times. This changing demand has considerably contributed to the need for automated approaches supporting employees in online customer service. Since automated approaches still struggle to meet the challenge to fully grasp the semantics of texts, hybrid approaches combining the complementary strengths of human and artificial intelligence show great potential for assisting employees. Against this background, the authors follow a design-oriented approach and develop an adapted textual Case-Based Reasoning (CBR) approach that integrates employees' feedback on semantic similarity. Using a real-world data set from the popular service website Quora, the authors demonstrate the practical applicability and evaluate their approach regarding performance in online customer service. Their novel approach surpasses humanbased, machine-based, and hybrid approaches in terms of effectiveness due to a refined retrieval of semantically similar customer problems. It is further superior in terms of efficiency, reducing the average time required to solve a customer problem.

The second paper, "How Perceptions of Intelligence and Anthropomorphism Affect Adoption of Personal Intelligent Agents" by Sara Moussawi, Raquel Benbunan-Fich and Marios Koufaris (2021), investigates to what extent users' perceptions of intelligence and perceptions of anthropomorphism influence their perceptions of usefulness, ease of use, enjoyment, initial trust, and intention to use. A personal intelligent agent (PIA) is a system that acts intelligently to assist a human using natural language. Examples include Siri and Alexa. These agents are powerful computer programs that operate autonomously and proactively, learn and adapt to change, react to the environment, complete tasks within a favorable timeframe, and communicate with the user using natural language to process commands and compose replies. PIAs are different from other systems previously explored in Information Systems (IS) due to their personalized, intelligent, and human-like behavior. Drawing on research in IS and Artificial Intelligence, the authors build and test a model of user adoption of PIAs leveraging their unique characteristics. Analysis of data collected from an interactive lab-based study for new PIA users confirms that both perceived intelligence and anthropomorphism are significant antecedents of PIA adoption. The findings contribute to the understanding of a quickly-changing and fast-growing set of technologies that extend users' capabilities.

The third paper, "Hybrid Intelligence in Hospitals: Towards a Research Agenda for Collaboration and TeamBuilding" by Milad Mirbabaie, Stefan Stieglitz and Nicholas R. J. Frick (2021), investigates how the implementation of hybrid intelligence affects the quality of organizational processes. Successful collaboration between clinicians is particularly relevant regarding the quality of the care process. In this context, the utilization of hybrid intelligence, such as conversational agents (CAs), is a reasonable approach for the coordination of diverse tasks. While there is a great deal of literature involving collaboration, little effort has been made to integrate previous findings and evaluate research when applying CAs in hospitals. By conducting an extended and systematic literature review and semi-structured expert interviews, the authors identified four major challenges and derived propositions where in-depth research is needed: 1 ) audience and interdependency; 2) connectivity and embodiment; 3) trust and transparency; and 4) security, privacy, and ethics. The results are helpful for researchers as we discuss directions for future research on CAs for collaboration in a hospital setting enhancing team performance. Practitioners will be able to understand which difficulties must be considered before the actual application of CAs.

Together, the three papers in this special issue represent important advances in the scientific discourse on HIS in business networks. We warmly recommend them to your reading. We would like to thank the editors of Electronic Markets to make this special issue possible and the reviewers of all submitted papers for their diligence in providing constructive feedback to the authors.

\section{References}

Attenberg, J., Ipeirotis, P., \& Provost, F. (2015). Beat the machine: challenging humans to find a predictive model's "unknown unknowns." Journal of Data and Information Quality (JDIQ), 6(1), 1. https://doi.org/10.1145/2700832.

Bothun, D., Liebermann, M. \& Rao, A. (2017). Bot.Me: a revolutionary partnership. How AI is pushing man and machine closer together. Consumer Intelligence Series $P w C$.

Card, D. \& Nelson, C. (2019). How automation and digital disruption are shaping the workforce of the future. Strategic HR Review, 18(6), 242-245. https://doi.org/10.1108/ SHR-08-2019-0067.

Cheng, J. \& Bernstein, M. S. (2015). Flock: hybrid crowd-machine learning classifiers. Proceedings of the 18th ACM Conference on Computer Supported Cooperative Work \& Social Computing. 600-611. https://doi.org/10.1145/2675133.2675214.

Dellermann, D., Ebel, P., Söllner, M. \& Leimeister, J. M. (2019a). Hybrid intelligence. Business \& Information Systems Engineering, 61. 1-7. https://doi.org/10.1007/s12599-019-00595-2.

Dellermann, D., Lipusch, N., Ebel, P., \& Leimeister, J. M. (2019b). Design principles for a hybrid intelligence decision support 
system for business model validation. Electronic Markets, 29(3), 423-441. https://doi.org/10.1007/s12525-018-0309-2.

Doroudi, S., Kamar, E., Brunskill, E. \& Horvitz, E. (2016). Toward a learning science for complex crowdsourcing tasks. Proceedings of the 2016 CHI Conference on Human Factors in Computing Systems, ACM. 2623-2634. https://doi.org/10.1145/2858036. 2858268.

Elshan, E. \& Ebel, P. (2020). Let's team up: designing conversational agents as teammates. Proceedings of the International Conference on Information Systems (ICIS).

Elshan, E., Engel, V. \& Ebel, P. (2021). Opening the black box of music royalties with the help of hybrid intelligence. Proceedings of the 54th Hawaii International Conference on System Sciences (HICSS). https://doi.org/10.24251/HICSS.2021.671.

Engel, C. T. \& Ebel, P. (2019). Data-driven service innovation: a systematic literature review and development of a research agenda. Proceedings of the 27th European Conference on Information Systems (ECIS), Stockholm \& Uppsala, Sweden. https://aisel.aisnet.org/ecis2019_rp/161.

Engel, C., Ebel, P. \& van Giffen, B. (2021a). Empirically exploring the cause-effect relationships of AI characteristics, project management challenges, and organizational change. 16th International Conference on Wirtschaftsinformatik (WI). https://aisel.aisnet.org/ wi2021/QDesign/Track10/3.

Engel, C., Elshan, E. \& Ebel, P. (2021b). Deploying a model for assessing cognitive automation use cases: insights from action research with a leading european manufacturing company. Proceedings of the 54th Hawaii International Conference on System Sciences (HICSS). https://doi.org/10.24251/HICSS.2021.755.

Feine, J., Gnewuch, U., Morana, S., \& Maedche, A. (2019). A taxonomy of social cues for conversational agents. International Journal of Human-Computer Studies, 132, 138-161. https://doi. org/10.1016/j.ijhcs.2019.07.009.

Følstad, A., \& Brandtzæg, P. B. (2017). Chatbots and the new world of HCI. Interactions, 24(4), 38-42. https://doi.org/10.1145/3085558.

Graef, R., Klier, M., Kluge, K., \& Zolitschka, J. F. (2021). Humanmachine collaboration in online customer service - a long-term feedback-based approach, Electronic Markets, 31(2). https://doi. org/10.1007/s12525-020-00420-9.

Ipeirotis, P. G. (2010). Analyzing the amazon mechanical turk marketplace. XRDS: Crossroads, The ACM Magazine for Students - Comp-YOU-Ter, 17(2), 16-21. https://doi.org/10.1145/18690 86.1869094

Janssen, J., \& Kirschner, P. A. (2020). Applying collaborative cognitive load theory to computer-supported collaborative learning: towards a research agenda. Educational Technology Research and Development, 68, 783-805. https://doi.org/10.1007/ s11423-019-09729-5.

Jordan, M. I., \& Mitchell, T. M. (2015). Machine learning: trends, perspectives, and prospects. Science, 349(6245), 255-260. https:// doi.org/10.1126/science.aaa8415.

Kamar, E. (2016a). Directions in hybrid intelligence: complementing AI Systems with human intelligence. Proceedings of the International Joint Conference on Artificial Intelligence.

Kamar, E. (2016b). Hybrid workplaces of the future. XRDS: Crossroads, the ACM Magazine for Students, 23(2), 22-25. https://doi. org/10.1145/3013488.

Kamar, E., Hacker, S. \& Horvitz, E. (2012). Combining human and machine intelligence in large-scale crowdsourcing. Proceedings of the 11th International Conference on Autonomous Agents and Multiagent Systems-Volume 1, International Foundation for Autonomous Agents and Multiagent Systems.

Kamar, E., Kapoor, A. \& Horvitz, E. (2015). Identifying and accounting for task-dependent bias in crowdsourcing. 3rd AAAI Conference on Human Computation and Crowdsourcing.
Kenney, M., \& Zysman, J. (2016). The rise of the platform economy. Issues in Science and Technology, 32(3), 61-69.

Knote, R., Janson, A., Söllner, M. \& Leimeister, J. M. (2019). Classifying smart personal assistants: an empirical cluster analysis. Proceedings of the 52nd Hawaii international conference on system sciences (HICSS). https://doi.org/10.24251/HICSS.2019.245.

Knote, R., Janson, A., Söllner, M., \& Leimeister, J. M. (2021). Value co-creation in smart services: a functional affordances perspective on smart personal assistants. Journal of the Association for Information Systems, 22(2), 5.

Kühl, N., Mühlthaler, M. \& Goutier, M. (2020). Supporting customeroriented marketing with artificial intelligence: automatically quantifying customer needs from social media. Electronic Markets, 30(3), 351-367. https://doi.org/10.1007/s12525-019-00351-0.

Lakkaraju, H., Kamar, E., Caruana, R. \& Horvitz, E. (2017). Identifying unknown unknowns in the open world: representations and policies for guided exploration. Proceedings of the AAAI Conference on Artificial Intelligence.

Lee, S.-s., Lee, J. \& Lee, K.-p. (2017). Designing intelligent assistant through user participations. Proceedings of the 2017 Conference on Designing Interactive Systems. 173-177. https://doi.org/10. 1145/3064663.3064733.

Maedche, A., Legner, C., Benlian, A., Berger, B., Gimpel, H., Hess, T., Hinz, O., Morana, S., \& Söllner, M. (2019). AI-based digital assistants. Business \& Information Systems Engineering, 61(4), 535-544. https://doi.org/10.1007/s12599-019-00600-8.

Mao, K., Yang, Y., Li, M. \& Harman, M. (2013). Pricing crowdsourcing-based software development tasks. Proceedings of the 26th International Conference on Software Engineering. 1205-1208. https://doi.org/10.1109/ICSE.2013.6606679.

Mirbabaie, M., Stieglitz, S., \& Frick, N. R. J. (2021) Hybrid intelligence in hospitals: towards a research agenda for collaboration and team-building, Electronic Markets, 31(2). https://doi.org/10. 1007/s12525-021-00457-4.

Mitchell, M., Bohus, D. \& Kamar, E. (2014). Crowdsourcing language generation templates for dialogue systems. Proceedings of the INLG and SIGDIAL 2014 Joint Session. 172-180. https://doi. org/10.3115/v1/W14-5003.

Moussawi, S., Benbunan-Fich, R., \& Koufaris, M. (2021). How perceptions of intelligence and anthropomorphism affect adoption of personal intelligent agents. Electronic Markets, 31(2). https://doi. org/10.1007/s12525-020-00411-w.

Neuhofer, B., Magnus, B. \& Celuch, K. (2020). The impact of artificial intelligence on event experiences: a scenario technique approach. Electronic Markets (2020). https://doi.org/10.1007/ s12525-020-00433-4.

Poser, M., \& Bittner, E. A. (2020). Hybrid teamwork: consideration of teamwork concepts to reach naturalistic interaction between humans and conversational agents. 15th International Conference on Wirtschaftsinformatik, Potsdam, Germany. https://doi.org/10. 30844/wi_2020_a6-poser.

Seeber, I., Bittner, E., Briggs, R. O., De Vreede, T., De Vreede, G.-J., Elkins, A., Maier, R., Merz, A. B., Oeste-Reiß, S., \& Randrup, N. (2020). Machines as teammates: a research agenda on AI in team collaboration. Information \& Management, 57(2), 103174. https:// doi.org/10.1016/j.im.2019.103174.

Stone, P., Brooks, R., Brynjolfsson, E., Calo, R., Etzioni, O., Hager, G., Hirschberg, J., Kalyanakrishnan, S., Kamar, E., \& Kraus, S. (2016). Artificial intelligence and life in 2030 - one hundred year study on artificial intelligence: Report of the 2015-2016 study panel. Analysis and Policy Observatory (APO). Stanford University Press. https://apo.org.au/node/210721.

Wambsganss, T., Niklaus, C., Cetto, M., Söllner, M., Handschuh, S. \& Leimeister, J. M. (2020). AL: an adaptive learning support system for argumentation skills. Proceedings of the 2020 CHI Conference 
on Human Factors in Computing Systems. 1-14. https://doi.org/ 10.1145/3313831.3376732.

Wambsganss, T., Küng, T., Matthias, S. \& Leimeister, J. M. (2021). Arguetutor: an adaptive dialog-based learning system for argumentation skills. Proceedings of the 2021 CHI conference on human factors in computing systems. 1-13. https://doi.org/10. 1145/3411764.3445781.

Wang, W. Y., Bohus, D., Kamar, E. \& Horvitz, E. (2012). Crowdsourcing the acquisition of natural language corpora: methods and observations. Proceedings of the IEEE Spoken Language Technology Workshop (SLT), IEEE. https://doi.org/10.1109/SLT. 2012.6424200.

World Economic Forum (2020). The future of jobs report 2020. Wef. http://www3.weforum.org/docs/WEF_Future_of_Jobs_ 2020.pdf.

Winkler, R., Elshan, E., Söllner, M. \& Leimeister, J. (2020a). SPAMA process model for developing smart personal assistants.
Proceeding of the Hawaiian International Conference on System Sciences (HICSS).

Winkler, R., Hobert, S., Salovaara, A., Söllner, M. \& Leimeister, J. M. (2020b). Sara, the lecturer: improving learning in online education with a scaffolding-based conversational agent. Proceedings of the 2020 CHI Conference on Human Factors in Computing Systems. 1-14. https://doi.org/10.1145/3313831.3376781.

Winkler, R., Söllner, M., \& Leimeister, J. M. (2021). Enhancing problem-solving skills with smart personal assistant technology. Computers \& Education, 165, 104148. https://doi.org/10.1016/j. compedu.2021.104148.

Zarkadakis, G., Jesuthasan, R., \& Malcolm, T. (2016). The 3 ways work can be automated. Harvard Business Review, 13, 2016.

Publisher's Note Springer Nature remains neutral with regard to jurisdictional claims in published maps and institutional affiliations. 